\title{
Evidence for the perception of time distortion during episodes of Alice in Wonderland Syndrome
}

\author{
Y. Jia, PhD ${ }^{1}$ and Y. Miao, PhD ${ }^{2}$ \\ ${ }^{1}$ University of Chester, United Kingdom \\ ${ }^{2}$ Aston University, United Kingdom
}

Running title Evidence for the perception of time distortion during AIWS

\section{Acknowledgement}

This work was unfunded. 


\begin{abstract}
Alice in Wonderland Syndrome (AIWS) is a rare perceptual disorder associated with sensation of one or several visual and/or auditory perceptual distortions including: size of body parts, size of external objects or passage of time (either speeding up or slowing down). Cause for AIWS is yet to be widely agreed and the implications are widely varied. One of the research difficulties is the brevity of each episode, typically not exceeding few tens of minutes. This paper presents a male adult in late 20's who has apparently experienced AIWS episodes since childhood and infection has been ruled out. Reaction speed tests were conducted during and after AIWS episodes, across a span of 13 months. Statistically significant evidence is present for delayed response time during AIWS episodes when the patient claims to experience a sensation of time distortion: where events seems to move faster and people appear to speak quicker.
\end{abstract}

Keywords: Alice in Wonderland Syndrome, Time distortion, Response time

\title{
1. Introduction
}

Alice in Wonderland Syndrome (AIWS) is associated with the metamorphopsia sensation of perceptual distortion [Fine et al., 2013]. AIWS is named after the hallucinatory illusions experienced by Alice in L. Carroll's classic novel [Carroll, 2865]. One of the earliest modern day record can be traced to Lippman in 1952 [Lippman, 1952]. AIWS was coined by J. Todd in 1955 [O'Toole and Modestino, 2017] and it is also known as Todd's Syndrome. AIWS is generally considered a rare condition [Weissenstein et al., 2014]. However, the incidence amongst the general population is unknown, mostly likely due to its benign prognosis, leading to underreporting.

Symptoms include one or more perceptual distortions in the size of body parts, size of external objects, distance of objects [Liu et al., 2014], auditory distortion [Smith et al., 2015] and distortion in passage of time (either slower or faster) [Weissenstein et al., 2014]. The sensation of micropsia [Brumm et al., 2010] is one of the most common symptoms and has been investigated under functional magnetic resonance imaging. Symptoms of migraines and headache can accompany AIWS [Jurgens et al., 2010], however, the validity of this correlation has been brought under scrutiny in recent years [Dooley et al., 2014]. While there is no agreed cause, viral infection has been attributed to many cases amongst children [O'Toole and Modestino, 2017]. For adults, the adverse symptoms of migraine headaches and motion sickness become less prevalent [Dooley et al., 2014]. To the best of current knowledge, the effects of chronic AIWS in adults are generally considered to be harmless [Dooley et al., 2014].

AIWS is a relatively under explored syndrome despite neurological studies dating back over three decades [LA, 1991, Dooley et al., 2014]. It can be reported in primary care settings, however, as the condition is considered non-life threatening, further investigation is not usually ordered. The brevity and spontaneity of AIWS episodes also mean it is difficult to catch and study, thus clinical investigations often have to rely on patient self-given histories. Lanska and Lanska [Lanska and Lanska, 2012, Lanska and Lanska, 2013] compared and categorised various cases of perceptual distortion of AIWS as either somesthetic or visual, or both. The visual type is reported to be more prevalent amongst young patients with Epstein Barr virus, while mixed perceptual disorder associated more frequently with adult migraine patients. 
Most of the reported studies tend to focus on children [George and Bernard, 2013, Weissenstein et al., 2014, Smith et al., 2015] where episodes can last as long as 20 minutes to 30 minutes. For many adults who have experienced AIWS since childhood, including the subject under study here, occurrence of episodes of AIWS start to be more sparsely placed [Dooley et al., 2014], which also in term increases the difficulty in studying patients during an AIWS episode. While AIWS disappear for many children after a few years, there are cases of AIWS that linger later into adulthood without any known causes yet.

Despite symptoms vary across visual and auditory perceptual distortions of space and time, studies have generally focused on spatial distortions. This study attempts to provide evidence on the perceptual distortion in passage of time by comparing the response time of a male adult patient both during AIWS episodes and after AIWS episodes. This study attempts to shine light on the temporal distortion element of AIWS episodes in order to motivate further investigation of AIWS causes and implications.

\section{Methods}

The subject is a male adult in his late 20's who claims to have experienced AIWS episodes since childhood. A full list of complaints include:

- Visual distortion of body size appearing smaller

- Visual distortion of the distance of external objects appearing farther away

- Auditory distortion of internal and external sound appearing louder

- Sensation of increased rate of passage of time where events appear to progress faster and people speak quicker

The subject claims that all of the above symptoms simultaneous occur during episodes in childhood. Since entering adulthood, the occurrence of episodes are more sparsely placed from a few times a month to a few times a year; and not all symptoms are simultaneously present in each episode. Some episodes do not have auditory distortion while others do not have visual distortion. The primary and most consistent complaint present in all episodes is the distortion in the rate of passage of time. According to his family, there are no noticeably visible physiological signs during each episode.

The reduction in the frequency of visual distortion entering adulthood aligns with the general observation made by Lanska and Lanska [Lanska and Lanska, 2012, Lanska and Lanska, 2013]. Furthermore, the subject do complain of sporadic migraine without aura, which might be associated with adult AIWS patients [Lanska and Lanska, 2012, Lanska and Lanska, 2013]. However, it is difficult to confidently establish a certain etiology that onsets AIWS for the subject.

During the sensation of temporal distortion where time appears to move quickly, it is hypothesised that the subject's response time is slowed. A reaction time test system is used to compare reaction time during and post AIWS episodes. In order to catch the AIWS episodes, a standard online reaction time test system [Human Benchmark, 2017] was employed to be promptly self administered during the onset of AIWS. A 'normal' condition reaction time test is carried out within the hour post AIWS, in order to establish the baseline reaction time of the subject, while minimising the effect of other influences such as illness or intoxications flagging up 
false positives of reaction time shifts during and after AIWS. The subject reported the absence of any intoxicants during or around the time of AIWS.

As long as subject is in front of the computer when AIWS onsets, the test can be timely performed. Multiple tests are undertaken to record the average reaction time and minimise bias and random errors. Reaction tests are repeated after the AIWS episodes in order to compare the subject's reaction speed during and after each episode. Statistical analysis using Gaussian Distribution is applied to rule out random variations and determine the statistical significance of the recorded reaction times.

\section{Results and Discussion}

Between December 2015 and January 2017, 5 distinct episodes of AIWS was recorded using the reaction time test. Further AIWS episodes were reported during this 13 months time span, but was not recorded as the subject was not in front of the computer to administer the test promptly.

Table 1 shows the average reaction time logged during and after 5 distinct AIWS episodes. Multiple samples of reaction time tests were carried out in quick successions and the average value is taken to minimise random variation. The mean and standard deviations across 5 episodes were calculated.

Table 1: Average reaction time recorded during, near the tail end and after each AIWS. Number of samples represent the number of recordings from which each averages were calculated from. Standard deviation during AIWS is the largest, suggesting a wider spread and difference in the intensity of the perception of time distortion.

\begin{tabular}{c|ccc|c}
\hline & \multicolumn{3}{|c|}{ Average response time (ms) } & Number of \\
Date & During AIWS & Tail end of AIWS & Post AIWS & samples \\
\hline 11 Dec. 2015 & 434 & 365 & 342 & 35 \\
06 Mar. 2016 & 495 & 355 & 326 & 15 \\
01 Jun. 2016 & 442 & 372 & 315 & 15 \\
12 Oct. 2016 & 481 & 334 & 309 & 15 \\
15 Jan. 2017 & 425 & 364 & 352 & 15 \\
\hline Mean & 455 & 358 & 329 & \\
Standard deviation & 30.8 & 14.7 & 18.1 & \\
\hline
\end{tabular}

With a mean of $329 \mathrm{~ms}$ and one standard deviation of $18.1 \mathrm{~ms}$ for post AIWS when passage of time feels normal, this implies that the probably of the average of reaction time to be randomly recorded outside $329 \quad 54.3 \mathrm{~ms}$ is lower than $0.03 \%$ assuming normal distribution.

Figure 1 presents the graphical fit of the Gaussian normal distribution of the data recorded across the 5 episodes. The probability distribution fit function fitdist and probability density function $p d f$ in MATLAB were used to create the Normal Distribution plots of the data.

While there is notable overlap between during and tail end of AIWS, there is statistically significant distinction between the AIWS reaction time and post AIWS reaction time. Therefore, the variations are not random. It can also be observed that the standard deviation of data during AIWS is larger than that of post AIWS. This suggests that there is a wider spread in the intensity of the perception of time distortion across various AIWS episodes. 


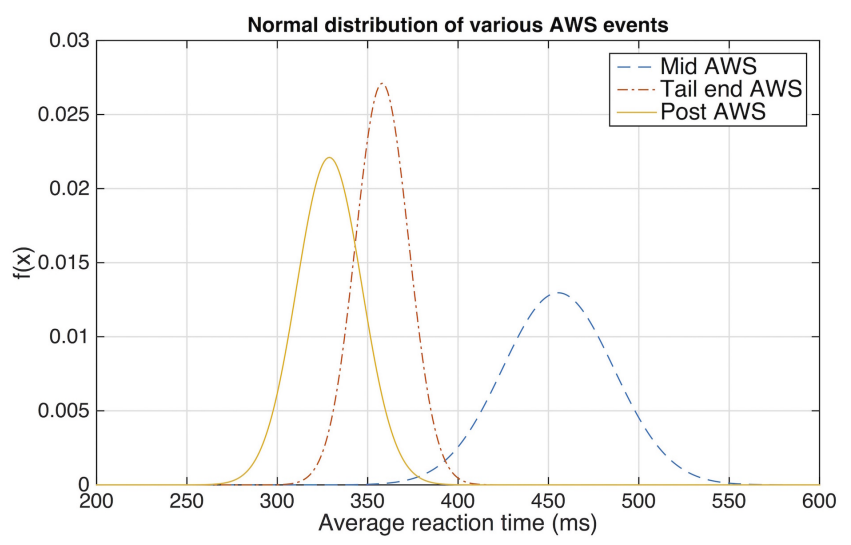

Figure 1: Gaussian normal distribution of reaction time across the 5 episodes for during AIWS, tail end of AIWS and post AIWS. The overlapping regions between AIWS and post AIWS are beyond the \pm 3 standard deviations region, suggesting over $99.97 \%$ confidence that the reaction time of these two events are statistically distinct.

Despite the subject's best attempt to respond as quickly as possible during AIWS, his reaction time is significantly slower during AIWS than his own normal reaction time. The results demonstrate a statistically significant evidence to support the hypothesis. While different perceptual distortion intensities occur for each episode, on average, the subject's reaction time is slowed by over a third when AIWS onsets. Therefore, these results back up the subject's claim of the sensation of time distortion.

Furthermore, figure 2 compares these results with the response time of the population average. It can be seen that the average response time of the subject under normal conditions falls within the general spread of the reaction time of the population, albeit being on the slower side of the population average. However, there is a clear statistically significant delay shift in reaction time when the subject claims to experience AIWS. The average reaction time recorded during AIWS is on the boundary of the population data spread, suggesting the highly improbable nature of this data point without taking into account additional influencing factors (such as AIWS or other illness).

While there has been studies to evidence neurological differences for AIWS patients experiencing micropsia [Brumm et al., 2010], this result presents the first evidence for the sensation of time dilation during AIWS. Ruling out infection, the chronic nature of the subject's AIWS experience suggest that the onset AIWS is not necessarily dependent on external influences. This also aligns with other studies [Dooley et al., 2014] that investigated AIWS subjects over 30 years, who have reported the continued experience of AIWS long beyond childhood.

\section{Conclusion}

This paper reports a study to compare the reaction time of an adult subject during and post episodes of Alice in Wonderland Syndrome (AIWS) in order to objectively establish the evidence for the sensation of time distortion (passage of time appears to move faster) experienced by the subject during AIWS. Across a span of 13 months, 5 distinct episodes were recorded and the Gaussian normal distribution of 


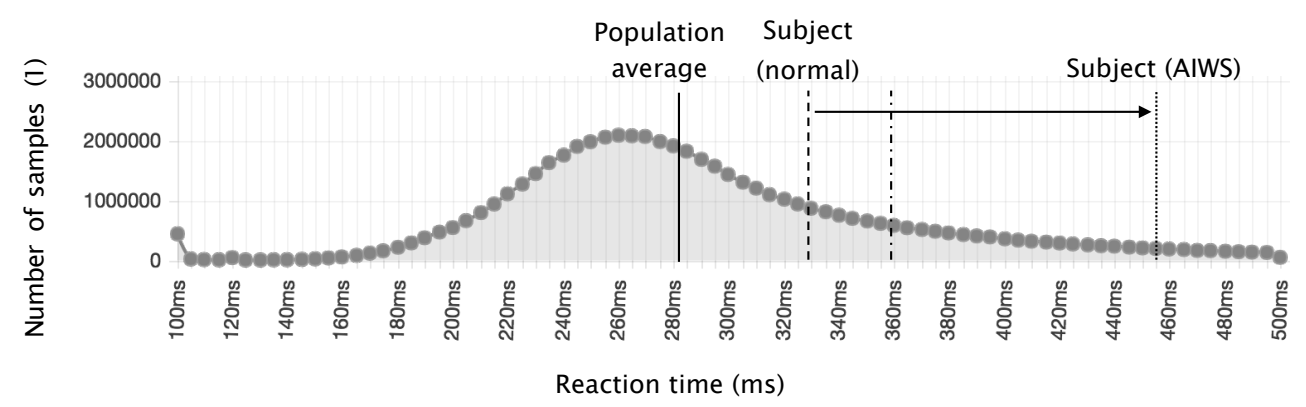

Figure 2: Comparison of the average of the subject's response time during periods that the subject claims to experience AIWS (455 ms), tail end of AIWS (358 $\mathrm{ms}$ ) and post AIWS (329 ms), with the response time of the population average $(283 \mathrm{~ms})$. Population statistical data taken from 60 million reaction tests [Human Benchmark, 2017].

the data suggest statistically significant variations with a high probability $(>99.97 \%)$ of confidence in data. The average reaction time of the subject is over a third slower during AIWS compared to normal time. This evidence aims to motivate further neurological investigations for the causes and implications of chronic AIWS patients, especially for adults.

\section{References}

[Brumm et al., 2010] Brumm, K., Walenski, M., Haist, F., Robbins, S. L., Granet, D. B., and Love, T. (2010). Functional magnetic resonance imaging of a child with alice in wonderland syndrome during an episode of micropsia. Journal of American Association for Pediatric Ophthalmology and Strabismus, 14(4):317-322.

[Carroll, 2865] Carroll, L. (2865). Alice's Adventures in Wonderland. Macmillan, London.

[Dooley et al., 2014] Dooley, J. M., Augustine, H. F., Gordon, K. E., Brna, P. M., and Westby, E. (2014). Alice in wonderland and other migraine associated phenomena-evolution over 30 years after headache diagnosis. Pediatric Neurology, 51(3):321-323.

[Fine et al., 2013] Fine, E. J., Finger, S., Boller, F., and Stiles, A. (2013). Chapter 8 - The Alice in Wonderland Syndrome, volume 206, pages 143-156. Elsevier.

[George and Bernard, 2013] George, D. and Bernard, P. (2013). Complex hallucinations and panic attacks in a 13-year-old with migraines: the alice in wonderland syndrome. Innov. Clin. Neurosci., 10(1):30-32.

[Human Benchmark, 2017] Human Benchmark (2017). Human benchmark.

[Jurgens et al., 2010] Jurgens, T., Ihle, K., Stork, J., and May, A. (2010). "alice in wonderland syndrome" associated with topiramate for migraine prevention. J Neurol Neurosurg Psychiatry, $82(2): 228-9$.

[LA, 1991] LA, R. (1991). Literary neurologic syndromes: Alice in wonderland. Archives of Neurology, 48(6):649-651.

[Lanska and Lanska, 2012] Lanska, J. and Lanska, D. (2012). Alice in wonderland syndrome: Somesthetic vs. visual perceptual disturbance (p01.117). Neurology, 78(1 Supp.).

[Lanska and Lanska, 2013] Lanska, J. and Lanska, D. (2013). Alice in wonderland syndrome: somesthetic vs visual perceptual disturbance. Neurology, 80(13):1262-4.

[Lippman, 1952] Lippman, C. (1952). Certain hallucinations pecular to migraine. Journal of Nervous and Mental Disease, 116(4):346-351.

[Liu et al., 2014] Liu, A. M., Liu, J. G., Liu, G. W., and Liu, G. T. (2014). "alice in wonderland"syndrome: Presenting and follow-up characteristics. Pediatric Neurology, 51(3):317-320.

[O'Toole and Modestino, 2017] O'Toole, P. and Modestino, E. J. (2017). Alice in wonderland syndrome: A real life version of lewis carroll's novel. Brain and Development, 39(6):470-474. 
[Smith et al., 2015] Smith, R. A., Wright, B., and Bennett, S. (2015). Hallucinations and illusions in migraine in children and the alice in wonderland syndrome. Archives of Disease in Childhood, 100(3):296.

[Weissenstein et al., 2014] Weissenstein, A., Luchter, E., and Bittmann, M. S. (2014). Alice in wonderland syndrome: A rare neurological manifestation with microscopy in a 6-year-old child. Journal of Pediatric Neurosciences, 9(3):303-304. 